\title{
Assessment of information included on the GOS 18 referral form used by optometrists
}

\author{
S. C. Lash \\ Southampton Eye Unit, Southampton General Hospital, Tremona Road, Southampton SO16 6YD, \\ UK
}

\begin{abstract}
Purpose: To audit the information included on GOS 18 forms used by UK optometrists when referring patients to an ophthalmologist.

Methods: All GOS 18 forms received in a hospital ophthalmology department over a 10-week period were photocopied and the categories of information presented were recorded.

Results: A total of 444 forms were analysed. The two most common referral categories were cataract $36.7 \%(n=163)$ and glaucoma 18.4\% $(n=82)$. Only $7 \%(n=11)$ of cataract referrals included details regarding effect on patient's lifestyle and willingness for surgery. Forty-seven per cent $(n=77)$ of referrals for cataract resulted in patients being listed for surgery. Eighty-two per cent ( $n=67$ ) of referrals for glaucoma included disc assessment, intraocular pressure and visual fields. Five per cent $(n=22)$ of optometrists gained the patients' consent for release of clinical information. Thirty-one per cent $(n=137)$ of forms had no practitioner name and $6 \%(n=27)$ gave no practice address.

Conclusion: Information included on GOS 18 forms could be improved with regard to cataract referrals. Feedback from ophthalmologists would be facilitated by inclusion of practitioner/practice details, and by completion of the consent section on the GOS 18.
\end{abstract}

Keywords: cataract, communication, consent, GOS 18, optometrist, referral

\section{Introduction}

Many patients are referred to the Hospital Eye Service by their optometrist either directly (Seward, 1999), or via their general medical practitioner. In view of the limited resources available, it is important that these referrals are necessary and timely. Referrals to ophthalmology departments have been analysed previously (Port and Pope, 1988; Brin and Griffen, 1995; Pooley and Frost, 1999), particularly in the area of glaucoma referrals (Tuck and Crick, 1991; Bell and O'Brien, 1997).

The current study investigates the completeness of information provided by the optometrist on the GOS 18 form and the common reasons for referral. Further

Received: 6 February 2002

Revised form: 5 August 2002

Accepted: 21 August 2002

Correspondence and reprint requests to: S. C. Lash.

Tel.: 02380794645 ; fax: 02380794120

E-mail address: stephenlash@hotmail.com analysis for cataract and glaucoma referrals is included. The issue of consent to release medical information back to the optometrist is addressed.

\section{Method}

All GOS 18 forms received in the Eye Unit at the Royal Bournemouth Hospital from 12 February to 23 April 2001 were photocopied and the presence of the following details recorded: practitioner name, practice address, date of birth, spectacle prescription, visual acuities, intraocular pressure (IOP), cup:disc (C:D) ratios, visual fields and patient consent. It should be emphasised that optometrists in the region were not aware that such specific analysis of their referrals was being undertaken.

The main reason for referral was recorded. Cataract referrals were further analysed as to inclusion of information regarding effect on patient's lifestyle and willingness for surgery. To assess the appropriateness of cataract referrals, the number of referrals resulting in surgery was determined by comparing the patients referred to a list of patients who had undergone cataract 
surgery since February 2001. This should be accurate as the waiting list for surgery is $3-4$ months and at the time of writing the latest referrals were received 9 months ago. Glaucoma referrals were analysed as to inclusion of information on C:D ratios, IOP and visual fields. No further analysis was carried out at this time.

\section{Results}

The total number of referrals over the 10 -week period was 444. The reasons for referral are summarised in Table 1.

With regard to cataract referrals, $7 \%(n=11)$ contained details regarding both an effect on the patient's lifestyle and willingness for surgery. A total of $36 \%$ $(n=59)$ recorded an effect on the patient's lifestyle and $11 \%(n=18)$ reported willingness for surgery. Fortyseven per cent $(n=77)$ were listed for cataract surgery as a result of referral.

Eighty-two per cent $(n=67)$ of glaucoma referrals included information on visual fields, C:D ratio and IOP; $89 \%(n=73)$ recorded C:D ratios, $97 \%(n=80)$ recorded IOP and $82 \%(n=67)$ recorded field information. No further analysis was performed at this time.

Field defect as the sole reason for referral contributed $9 \%(n=36)$ of referrals. Nearly $30 \%$ of these came from one practice. No further analysis was performed at this time.

Five per cent $(n=22)$ of optometrists gained the patients' consent with the patient signing the GOS 18 form to permit release of medical information to the optometrist (Table 2). Thirty-one per cent $(n=137)$ of forms had no legible optometrist name and $6 \%(n=27)$ gave no practice address.

\section{Discussion}

As with other studies (Port and Pope, 1988; Brin and Griffen, 1995; Pooley and Frost, 1999) cataract and glaucoma are the most common reasons for referral. It was interesting to note that significant numbers were

Table 1. The reasons for referral recorded on the GOS 18 forms

\begin{tabular}{rrl}
\hline No. of referrals & Percentage & Reason for referral \\
\hline 163 & 36.7 & Cataract \\
82 & 18.4 & Glaucoma \\
38 & 8.6 & Macular changes \\
36 & 8.1 & Field defect \\
16 & 3.6 & Retinal lesion \\
16 & 3.6 & Low vision aid assessment \\
16 & 3.6 & Capsular thickening \\
12 & 2.7 & Orthoptic assessment \\
6 & 1.4 & Flashes and floaters \\
59 & 13.3 & Others \\
\hline
\end{tabular}

Table 2. Information on GOS 18 forms

\begin{tabular}{lcl}
\hline $\begin{array}{l}\text { No. of } \\
\text { referrals }\end{array}$ & $\begin{array}{l}\text { Percentage of } \\
\text { patients }\end{array}$ & $\begin{array}{l}\text { Information } \\
\text { recorded on GOS 18 }\end{array}$ \\
\hline 22 & 5.0 & Obtained patients' consent \\
307 & 69.1 & Practitioner name recorded \\
417 & 93.9 & Practice address recorded \\
355 & 80.0 & Patients' date of birth recorded \\
404 & 91.0 & Spectacle prescription recorded \\
\hline
\end{tabular}

also referred with macular changes $8.6 \%(n=38)$, field defects $8.1 \%(n=36)$ and retinal lesions $3.6 \%(n=16)$.

The GOS 18 is a generic form which does not prompt the optometrist to ascertain specific, relevant information. Few cataract referrals $(7 \%)$ contained full information as recommended in the Action on Cataracts document (NHS Executive, 2000). It is common experience for patients referred with cataract to be seen in clinic not knowing why they have been referred and/or not realising they have a choice with regard to surgery. Some patients may not be unduly troubled by their cataracts, and some might not want surgery despite an effect on their lifestyle. A study of cataract referrals in the West Midlands found that patients were sometimes referred too soon and that more information on lifestyle effects would help in prioritising referrals (Latham and Misson, 1997). Of the patients referred in our audit, $47 \%$ were listed for surgery. The accuracy of optometrist referrals is not in doubt, this suggests that the timing and appropriateness of referral could be improved. One approach to reduce some of these unnecessary referrals is to have a specific referral form for cataracts. A disadvantage of this system is that it requires another form which increases paperwork. To extrapolate, it would not be useful to have specific referral forms for every condition. However, with such large numbers of patients referred with cataract, a specific form in this area may reduce a significant number of unnecessary referrals. Another way of improving these referrals is for ophthalmologists to communicate to optometrists the need for more specific questioning of patients to assess suitability for referral for cataract.

Most glaucoma referrals (82\%) included details on C:D ratios, IOP and visual fields. Using a combination of all three investigations has been shown to have the highest positive predictive value (Bell and O'Brien, 1997; Newman et al., 1998; Harper and Reeves, 1999; Theodossiades and Murdoch, 1999). Glaucoma referrals pose a problem as highlighted in a recent audit (Vernon and Ghosh, 2001), which found that locally agreed guidelines for referral did not improve the true positive referral rate for glaucoma. It has been suggested that any abnormal results should be repeated to reduce false positive rates: in their study Bell and O'Brien (1997) found that visual field tests were least likely to have been 
conducted a second time. Recent College of Optometrists guidelines on repetition of field tests (College of Optometrists, 2001) prompted a response from the Association of Optometrists that stated there was no legal obligation to repeat field tests and repetition did not constitute part of the sight test unless there was a specific local initiative to do so. There is currently much debate regarding what constitutes a sight test and whether repeating investigations should be funded within the basic sight test. Without local initiative, with or without funding, field defects, mostly detected by non-optometric staff as part of 'pre-screening', are likely to continue to be referred in significant numbers.

Few optometrists $(5 \%)$ gained the patients' consent for release of medical information back to the optometrist. This is similar to the finding of Whittaker et al. (1999), who found only 17/107 GOS 18 forms they examined to contain consent, but suggested that the ophthalmologist is equally capable of gaining the patients' consent. The importance of communication between the two professions has been emphasised recently (Ingram and Culham, 2001). This can be facilitated by ensuring that the section for consent included on the GOS 18 is completed. It is also important that ophthalmologists should be encouraged to reply to all referrals. A recent internal audit of replies to optometrist referrals in our department revealed reply rates of $0-60 \%$ for different consultant clinics (unpublished data): Whittaker et al. (1999) found that ophthalmologists replied to optometrists in $17 / 107$ cases $(2 / 17$ where the optometrist had gained the consent and 15/90 where they had not).

\section{Conclusion}

The GOS 18 is a generic form for referral and, as such, has obvious limitations. Cataracts constitute one-third of referrals and reduction in unnecessary referrals in this area would be beneficial. Specific referral forms for cataract might reduce unnecessary referrals, but educating optometrists to obtain relevant information regarding effect on lifestyle and willingness for surgery may reduce unnecessary referrals without increasing paperwork.

Glaucoma referrals are problematic and it may be difficult to improve the number of true positive referrals given the epidemiology and early diagnostic uncertainties of glaucoma. However, optometrists should be encouraged to examine and report on the discs, IOPs and fields, and to repeat tests where possible.

Ophthalmologists need to reply to all optometrist referrals. Communication between the ophthalmologist and the optometrist is essential. The optometrist can make a positive contribution by ensuring that the patient signs the consent section of the GOS 18, and by including the optometrists' name and practice address in all referrals.

\section{References}

Bell, R. W. D. and O'Brien, C. (1997) Accuracy of referral to a glaucoma clinic. Ophthal. Physiol. Opt. 17, 7-11.

Brin, B. N. and Griffen, J. R. (1995) Referrals by optometrists to ophthalmologist and other providers. J. Am. Optom. Assoc. 66, 154-159.

College of Optometrists (2001) Framework for optometric referrals.

Harper, R. A. and Reeves, B. C. (1999) Glaucoma screening: the importance of combining test data. Optom. Vis. Sci. 76, 537-543.

Ingram, D. V. and Culham, L. E. (2001) Ophthalmologists and optometrists - interesting times? Br. J. Ophthalmol. 85, 769770.

Latham, K. and Misson, G. (1997) Patterns of cataract referral in the West Midlands. Ophthal. Physiol. Opt. 17, 300-306.

Newman, D. K., Anwar, S. and Jordan, K. (1998) Glaucoma screening by optometrists: positive predictive value of visual field testing. Eye 12, 921-924.

NHS Executive (2000) Action on Cataracts. Good practice guidance. P.O Box 777, London, SE1 6XH: DOH,27.

Pooley, J. E. and. Frost, E. C. (1999) Optometrists' referrals to the Hospital Eye Service. Ophthal. Physiol. Opt. 19, S16S24.

Port, M. J. and Pope, C. A. (1988) Referrals and notifications by British optometrists. Ophthal. Physiol. Opt. 8, 323-326.

Seward, H. (1999) New Cataract clinic. Direct referral from optometrists. Mayday Healthcare and Croydon PCG.

Theodossiades, J. and Murdoch, I. (1999) Positive predictive value of optometrist-initiated referrals for glaucoma. Ophthal. Physiol. Opt. 19, 62-67.

Tuck, M. W. and Crick, R. P. (1991) Efficiency of referral for suspected glaucoma. BMJ 302, 998-1000.

Vernon, S. A. and Ghosh, G. (2001) Do locally agreed guidelines for optometrists concerning the referral of glaucoma suspects influence referral practice? Eye 15, 458-463.

Whittaker, K. W., Ikram, K., Anderson, D. F., Kiel, A. W. and Luff, A. J. (1999) Non-communication between ophthalmologists and optometrists. J. R. Soc. Med. 92, 247-248. 